\title{
Nonradial pulsations in classical Cepheids of the Magellanic Clouds
}

\author{
Pawel Moskalik \\ Copernicus Astronomical Center, ul. Bartycka 18, 00-716 Warsaw, \\ Poland \\ Zbigniew Kołaczkowski \\ Wroctaw University Observatory, ul. Kopernika 11, 51-622 Wroctaw, \\ Poland \\ Tomasz Mizerski \\ Warsaw University Observatory, Al. Ujazdowskie 4, 00-478 Warsaw, \\ Poland
}

\begin{abstract}
We have performed systematic frequency analysis of the LMC Cepheids observed by OGLE project. Several new types of pulsation behaviour are identified, including triple-mode and amplitude-modulated double-mode pulsations. In $\sim 10 \%$ of the first overtone Cepheids we find low amplitude secondary periodicities corresponding to nonradial modes. This is the first evidence for excitation of nonradial oscillations in Classical Cepheid variables.
\end{abstract}

\section{Introduction}

For decades Cepheids and RR Lyrae stars have been considered primary examples of purely radial pulsators. This simple picture has changed in recent years, when nonradial modes have been detected in a substantial fraction of RR Lyrae variables in various stellar systems (Kovács 2002; Moskalik \& Poretti 2003; Alcock et al. 2003). Motivated by these findings, we have undertaken a systematic search for nonradial pulsators among Pop. I Cepheids of the Magellanic Clouds. In this paper we present our results for the LMC, with particular emphasis on the new types of pulsation behaviour detected during the survey. The analysis of the SMC Cepheids is currently underway and will be presented at a later date.

\section{Search for multiperiodicity}

The primary source of data for our analysis is the OGLE-II photometry obtained with the Difference Image Analysis method (Żebruń et al. 2001). This photometry covers the time base of $1000-1200 \mathrm{~d}$, with $250-500 \mathrm{I}$-band flux measurements per star. Such a long and uniform dataset is particularly well suited for our task. The search for multiperiodicity is conducted for all LMC Cepheids 
identified by the OGLE team (Udalski et al. 1999; Soszyński et al. 2000), except objects marked in their catalogue as FA, which are mostly Pop. II variables. The search is performed with a standard consecutive prewhitening technique. First, the data are least square fitted with the single frequency Fourier sum of the form

$$
I(t)=\langle I\rangle+\sum_{k} \mathrm{~A}_{k} \cos \left(2 \pi k \mathrm{f}_{0} t+\phi_{k}\right)
$$

with pulsation frequency $\mathrm{f}_{0}$ also being optimised. The Fourier transform of the residuals of the fit is then calculated over the range of $0-5 \mathrm{~d}^{-1}$, in order to reveal any secondary periodicities. In the next step, a new Fourier fit with all frequencies identified so far and their linear combinations is performed and the residuals to the fit are searched for additional periodicities again. The process is repeated until no new frequencies appear. The most interesting results of our survey are summarised below.

\section{FO/SO/TO triple-mode Cepheids}

In two of the previously known first/second overtone (FO/SO) double-mode Cepheids a third strong periodicity is detected. The stars are listed in Table 1. The period ratio of $P_{3} / P_{2} \simeq 0.84$ identifies the new mode as a third radial overtone. With three radial modes observed, these stars will strongly constrain the Cepheid evolutionary tracks. Preliminary calculations show already that both objects are on the first crossing of the instability strip (Dziembowski, private communication).

Table 1. Triple-mode Cepheids

\begin{tabular}{cccccc}
\hline Star & $\mathrm{P}_{1}[\mathrm{~d}]$ & $\mathrm{P}_{2} / \mathrm{P}_{1}$ & $\mathrm{P}_{3} / \mathrm{P}_{2}$ & $\mathrm{~A}_{2} / \mathrm{A}_{1}$ & $\mathrm{~A}_{3} / \mathrm{A}_{1}$ \\
\hline LMC SC3-360128 & 0.54128 & 0.8056 & 0.8400 & 0.167 & 0.311 \\
LMC SC5-338399 & 0.57951 & 0.8052 & 0.8403 & 0.111 & 0.178 \\
\hline \hline
\end{tabular}

\section{FO/SO double-mode Cepheids with amplitude modulation}

In 18 out of $55 \mathrm{FO} / \mathrm{SO}$ double-mode Cepheids we detect residual signal in the vicinity of primary pulsation frequencies. With the OGLE-II data these secondary peaks are always unresolved from the primary ones. Therefore, we repeat the analysis with the MACHO data (Allsman \& Axelrod 2001), which is of lower quality, but provides a much longer time base $(\sim 2700 \mathrm{~d})$. In 8 stars we are now able to resolve the secondary power into two peaks located on opposite sides of the primary (radial) peak. Together with the primary frequency they form a close equidistant triplet. A typical example of such a pattern is displayed in Fig. 1. The triplet structure can appear either around one (3 stars) or around both radial modes ( 5 stars). In one object we detect a triplet and an equally spaced quintuplet. Weak evidence of a quintuplet structure is also noticeable in 

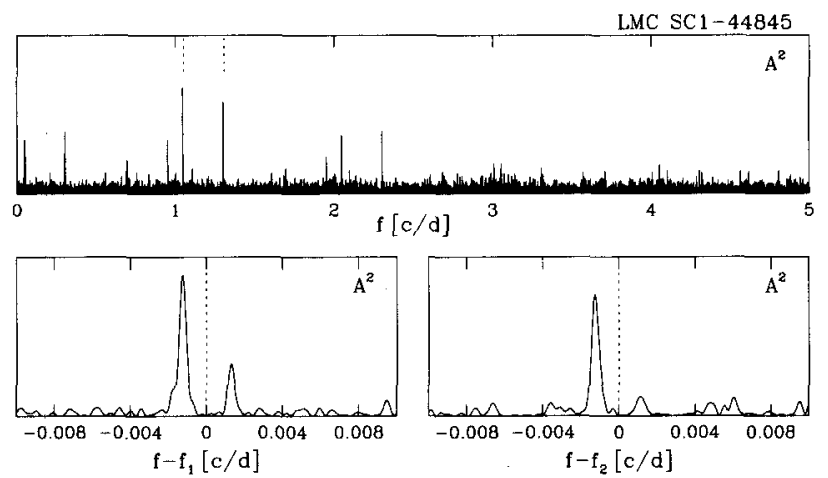

Figure 1. Power spectrum of FO/SO double-mode Cepheid LMC SC1-44845 after prewhitening with two radial frequencies and their linear combinations. Lower panels display the fine structure around the radial modes. The frequencies of the (removed) radial modes are indicated by the vertical dashed lines.

two other stars. In all stars the amplitudes of the side peaks are below 0.03 . The separation of components is always very small and corresponds to the beat period of more than $750 \mathrm{~d}$. When present around both radial modes, the two triplets/quintuplets have identical frequency spacings.

One more star can be resolved with MACHO data, and instead of triplets we find only doublets. In this particular object the secondary peaks are detected at very low signal-to-noise ratio. Therefore, it is likely that the detected doublets are in fact part of triplets, with the third component being buried in the noise.

The triplet/quintuplet frequency structures discovered in the FO/SO doublemode Cepheids can result from excitation of nonradial modes (Nowakowski \& Dziembowski 2001), but they can also be produced by periodic amplitude and phase modulation of the radial modes. The latter hypothesis seems to be more likely, as it can naturally explain why the frequency splittings of both radial modes are always the same.

\section{Nonradial pulsators}

In 46 first overtone Cepheids we detect well resolved secondary peaks close to the dominant radial mode. Two examples of such a behaviour are shown in Fig. 2. In most stars only one peak is present, but in several cases two or even three peaks are found, usually on the same side of the primary frequency. The observed patterns cannot be explained by amplitude and/or phase modulation. The period ratios are incompatible with those of radial modes, implying that the newly detected periodicities must correspond to nonradial modes of oscillation.

The secondary peaks are always very small. Their amplitudes are $10-30$ times lower than the amplitude of the radial mode. In most cases nonradial modes are found on the low frequency side of the dominant peak. The frequency separation $\Delta f=f-f_{0}$ is typically between $-0.15 \mathrm{~d}^{-1}$ and $+0.05 \mathrm{~d}^{-1}$. This is 



Figure 2. Power spectrum of two first overtone Cepheids after prewhitening with the primary frequency and its harmonics. The right column of the plot displays the fine structure in the vicinity of the radial mode. The frequency of the (removed) radial mode is indicated by the vertical dashed line.

very similar to $\Delta \mathrm{f}$ range found for the nonradially pulsating RRc stars (Alcock et al. 2000).

Forty-six stars in which nonradial modes are detected constitute $10 \%$ of the LMC first overtone Cepheid sample. In sharp contrast, nonradial modes are not found in any of the 720 fundamental mode Cepheids, with a possible exception of LMC SC17-157908, where marginal detection can be claimed. Clearly, the incidence rate of nonradial pulsations is dramatically lower in the fundamental mode variables.

Nonradial modes are also found close to the first overtone in two of the fundamental/first overtone double-mode Cepheids. In both stars, however, the secondary peak is only weakly significant and requires confirmation with better data.

Acknowledgments. This work has been supported by Polish KBN grants 5 P03D 01220 and 5 P03D 03020.

\section{References}

Alcock, C., Allsman, R., Alves, D.R., et al. 2000, ApJ, 542, 257

Alcock, C., Alves, D.R., Becker, A., et al. 2003, ApJ, 598, 597

Allsman, R.A., Axelrod, T.S. 2001, astro-ph/0108444

Kovács, G. 2002, in ASP Conf. Ser., Vol. 259, Radial and Nonradial Pulsations as Probes of Stellar Physics, eds C. Aerts, T.R. Bedding \& J. ChristensenDalsgaard (San Francisco: ASP), 396

Moskalik, P., Poretti, E. 2003, A\&A, 398, 213

Nowakowski, R.M., Dziembowski, W.A. 2001, Acta Astron., 51, 5

Soszyński, I., Udalski, A., Szymański, M., et al. 2000, Acta Astron., 50, 451

Udalski, A., Soszyński, I., Szymański, M., et al. 1999, Acta Astron., 49, 223

Żebruń, K., Soszyński, I., Woźniak, P.R. et al. 2001, Acta Astron., 51, 317 